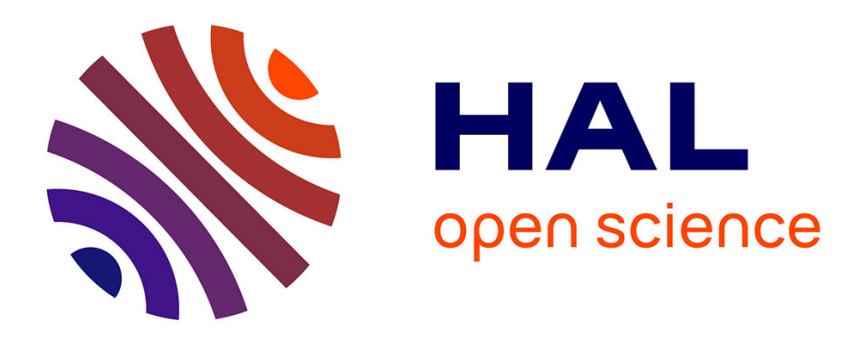

\title{
Mesure de la viscosité des semiconducteurs liquides
}

\author{
J.F. Rialland, J.C. Perron
}

\section{To cite this version:}

J.F. Rialland, J.C. Perron. Mesure de la viscosité des semiconducteurs liquides. Revue de Physique Appliquée, 1976, 11 (2), pp.263-269. 10.1051/rphysap:01976001102026300 . jpa-00244056

\section{HAL Id: jpa-00244056 https://hal.science/jpa-00244056}

Submitted on 1 Jan 1976

HAL is a multi-disciplinary open access archive for the deposit and dissemination of scientific research documents, whether they are published or not. The documents may come from teaching and research institutions in France or abroad, or from public or private research centers.
L'archive ouverte pluridisciplinaire HAL, est destinée au dépôt et à la diffusion de documents scientifiques de niveau recherche, publiés ou non, émanant des établissements d'enseignement et de recherche français ou étrangers, des laboratoires publics ou privés. 
Classification

Physics Abstracts

7.620

\title{
MESURE DE LA VISCOSITÉ DES SEMICONDUCTEURS LIQUIDES
}

\author{
J. F. RIALLAND et J. C. PERRON
}

Laboratoire de Génie Electrique (L. G. E. P.-L. C. I. E.),

33, avenue du Général Leclerc, 92260, Fontenay-aux-Roses, France

(Reçu le 30 juin 1975, révisé le 12 septembre 1975, accepté le 2 octobre 1975)

\begin{abstract}
Résumé. - Nous décrivons dans cet article une méthode de mesure de la viscosité dynamique des liquides semiconducteurs. Le viscosimètre rotatif que nous avons mis au point permet de mesurer des viscosités comprises entre 0,05 et $1000 \mathrm{P}$ jusqu'à des températures de $800^{\circ} \mathrm{C}$. Dans un premier temps nous avons testé notre dispositif avec le sélénium. Nous avons vérifié que le sélénium se comporte comme un liquide newtonien. La courbe de la viscosité en fonction de l'inverse de la température absolue tracée en coordonnées semilogarithmiques présente deux singularités à 280 et $500^{\circ} \mathrm{C}$. Le fait d'avoir contrôlé l'écoulement nous permet de les associer à des variations structurales du liquide et non pas à l'établissement de turbulences.
\end{abstract}

Abstract. - In this paper a method is reported for measuring the dynamic viscosity of liquid semiconductors. With the rotating cylinder viscometer described here, viscosity can be determined between 0.05 and $1000 \mathrm{P}$ for temperatures up to $800^{\circ} \mathrm{C}$. As a first experiment, this method has been successfully applied to liquid selenium. It has been possible to verify that selenium is a newtonian liquid. The curve of logarithm of viscosity versus the reciprocal absolute temperature displays two singularities at 280 and $500^{\circ} \mathrm{C}$. In the measurements the flow has always been laminar; so the anomalies are probably connected with changes in structure of the liquid and not with the occurrence of turbulence.

1. Introduction. - De nombreuses études effectuées sur les alliages sélénium-tellure ont mis en évidence l'existence de chaînes et d'anneaux (ou pseudoanneaux) dans la phase liquide. La viscosité, paramètre associé à la dynamique du liquide soumis à des contraintes, peut apporter des précisions quant à l'évolution de la structure en fonction de la température et du titre de l'alliage.

Dans ce but, nous avons mis au point un viscosimètre rotatif qui permet de mesurer des viscosités comprises entre 0,05 et $1000 \mathrm{P}$ jusqu'à des températures de $800^{\circ} \mathrm{C}$. L'intérêt des viscosimètres rotatifs est qu'en régime laminaire s'établit un écoulement de Couette. Le profil des vitesses est alors indépendant de la viscosité du liquide étudié. De plus, lorsque le rayon du cylindre intérieur est voisin de celui du cylindre extérieur, le profil est pratiquement linéaire. En faisant varier la vitesse de rotation relative des deux cylindres, on obtient l'évolution de la viscosité en fonction du gradient de vitesse ; on peut ainsi vérifier si le liquide est newtonien ou non. Enfin, ce type de viscosimètres permet de contrôler que la mesure est effectuée alors que l'écoulement est laminaire.

Dans le cas des alliages sélénium-tellure, les mesures sont rendues plus difficiles du fait de leur pression de vapeur élevée et de leur grande activité chimique. Dans cet article sont décrits la méthode retenue, sa mise en œuvre et les résultats obtenus pour le sélénium.
2. Principe. - Le liquide dont on veut déterminer la viscosité est placé entre deux cylindres coaxiaux. Lorsque le cylindre intérieur (rotor) est entraîné à une vitesse angulaire constante $\Omega_{1}$ et le cylindre extérieur (stator) maintenu fixe, la vitesse linéaire du liquide à la distance $r$ de l'axe est donnée, tant que l'écoulement est laminaire par [1] :

$$
v(r)=\frac{\Omega_{1} R_{1}^{2}}{R_{2}^{2}-R_{1}^{2}} \frac{R_{2}^{2}-r^{2}}{r}
$$

où $R_{2}$ est le rayon du stator et $R_{1}$ celui du rotor.

$\mathrm{Si}$ le liquide est newtonien, le moment des forces exercées sur le rotor s'écrit [1]

$$
C=-\frac{4 \pi \eta \Omega_{1} R_{1}^{2} R_{2}^{2}}{R_{2}^{2}-R_{1}^{2}} h
$$

où $h$ est la hauteur de liquide entre les deux cylindres et $\eta$ la viscosité dynamique.

En régime stationnaire, le couple moteur exercé sur le rotor du viscosimètre doit être égal à $-C$.

D'après l'éq. (2), on constate que pour une viscosité donnée le couple est proportionnel à la vitesse de rotation du rotor. Lorsque ceci n'est pas vérifié soit le liquide n'est pas newtonien, soit l'écoulement est turbulent. Ces deux cas peuvent être différenciés. En effet la turbulence apparaît pour une vitesse critique au-dessus de laquelle la relation (2) n'est plus vérifiée. 
Au contraire, si le liquide n'est pas newtonien, la relation (2) n'est jamais vérifiée. L'enregistrement du couple en fonction de la vitesse permet ainsi de préciser le cas dans lequel on se trouve.

L'intérêt de la méthode retenue est double. Comme nous venons de le voir elle permet tout d'abord de savoir si le liquide étudié est newtonien ou non. Lorsque le liquide n'est pas newtonien, il est possible d'associer une viscosité apparente caractéristique du gradient de vitesse imposé (ou de la contrainte exercée). En effet, en choisissant un viscosimètre tel que l'entrefer $R_{2}-R_{1}$ soit petit devant $R_{2}$ (ou $R_{1}$ ), l'éq. (1) peut se mettre sous la forme :

$$
v=\Omega_{1} R_{1} \frac{R_{2}-r}{R_{2}-R_{1}} .
$$

Le profil de vitesse est alors linéaire et le gradient de vitesse constant. Le couple mesuré et la viscosité qui s'en déduit peuvent ainsi être déterminés en fonction du gradient de vitesse.

Cette méthode permet de vérifier que les mesures sont effectuées alors que l'écoulement est laminaire. Considérons le cas d'un liquide newtonien. Tant que la vitesse de rotation du rotor est inférieure à une vitesse critique $\Omega_{\mathrm{c}}$, l'écoulement est laminaire et le couple mesuré est proportionnel à la vitesse de rotation. Au-delà de cette vitesse critique, le couple n'est plus proportionnel à $\Omega_{1}$. La stabilité des écoulements de Couette a principalement été étudiée par Rayleigh [2] et Taylor [3]. Lorsque le fluide est sans viscosité, la condition de stabilité s'écrit :

$$
\Omega_{2} R_{2}^{2}>\Omega_{1} R_{1}^{2}
$$

où $\Omega_{2}$ est la vitesse de rotation du cylindre extérieur et $\Omega_{1}$ celle du cylindre intérieur.

Cette inégalité est établie en supposant que les deux cylindres tournent dans le même sens. Dans notre cas, le cylindre extérieur étant immobile, l'inégalité (4) n'est jamais vérifiée. Cependant, l'existence de forces visqueuses de frottement au sein du fluide entraîne que l'écoulement peut être stable tant que le nombre de Taylor (ou de Reynolds) reste inférieur à une valeur critique. Lorsque l'entrefer $R_{2}-R_{1}$ est petit devant $R_{2}$, le nombre de Taylor de l'écoulement est donné par l'expression, [4] :

$$
T=\frac{4 R_{1}^{2}\left(R_{2}-R_{1}\right)^{4}}{R_{2}^{2}-R_{1}^{2}}\left(\frac{\Omega_{1}}{v}\right)^{2}
$$

où $v$ représente la viscosité cinématique du liquide. La valeur critique $T_{\mathrm{c}}$ vaut environ 3400 [4]. Tant que le nombre de Taylor reste inférieur à $T_{\mathrm{c}}$, l'écoulement peut être stable. Ceci signifie que toute fluctuation de vitesse est amortie au cours du temps. Les temps de stabilisation sont d'autant plus grands que le nombre de Taylor est proche de $T_{\mathrm{c}}$. Les calculs des limites de stabilité sont réalisés en supposant que les fluctuations sont faibles (ce qui permet de linéariser les équations).
La théorie ne permet pas de prévoir la réponse aux fortes perturbations. Il est possible qu'un écoulement stable par rapport à de petites fluctuations devienne instable par rapport à de plus grandes [1].

Dans tous les cas, la théorie ainsi que l'expérience montrent que l'écoulement est plus stable lorsque le cylindre extérieur tourne. Cependant, d'un point de vue mécanique, cette solution est plus difficile à mettre en œuvre. Aussi avons-nous choisi de faire tourner le cylindre intérieur et de maintenir fixe le cylindre extérieur.

3. Description du viscosimètre. - Le viscosimètre est constitué principalement de deux cylindres coaxiaux (Fig. 1). Le cylindre intérieur de rayon $R_{1}$ et de

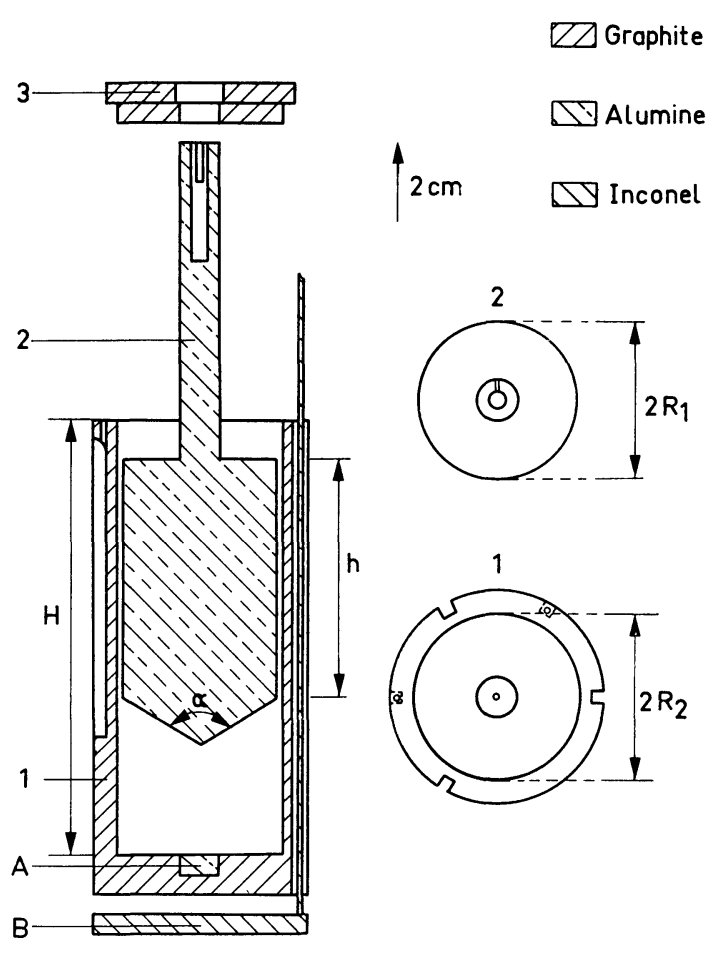

Fig. 1. - Viscosimètre. 1) Stator. 2) Rotor. 3) Bouchon. A) Pastille de centrage. B) Plateau du viscosimètre.

hauteur $h$ constitue le rotor ; il est prolongé à sa partie inférieure par un cône de révolution et à sa partie supérieure par un arbre cylindrique. Le sommet du cône se loge dans un trou de centrage percé au fond du stator. Un alésage réalisé à l'extrémité de l'arbre permet de rendre solidaires par l'intermédiaire d'un axe muni d'un ergot le rotor et le moteur d'entraînement (Fig. 1, 2 et 3).

Le cylindre extérieur constitue le stator ; son rayon intérieur est $R_{2}$. A la périphérie du stator sont pratiquées cinq rainures dans lesquelles sont logés deux thermocouples de mesure et trois tiges reliées à un plateau en inconel sur lequel prend place le viscosimètre. Le dispositif composé du plateau et des tiges 


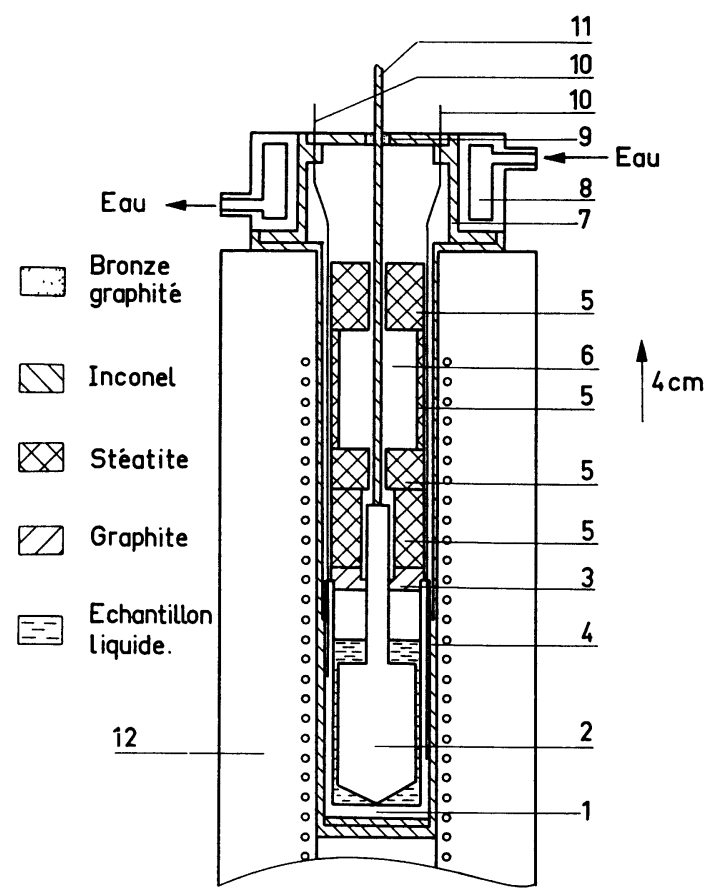

Fig. 2. - Dispositif expérimental. 1) Stator. 2) Rotor. 3) Bouchon du viscosimètre. 4) Enceinte. 5) Bouchon thermique. 6) Chambre de condensation. 7) Chapeau de fermeture. 8) Chemise d'eau. 9) Bague de centrage. 10) Thermocouple. 11) Axe de transmission. 12) Four.

permet d'extraire le viscosimètre de l'enceinte (Fig. 1 et 2).

Le viscosimètre comprend également un bouchon en graphite percé d'un alésage qui permet le centrage de l'arbre du rotor (et donc du rotor) par rapport au stator (Fig. 1). Par ailleurs, le sélénium et le tellure ont des pressions de vapeur élevées. Aussi, ce bouchon a également pour rôle de ralentir la diffusion des vapeurs dans l'enceinte supérieure.

Nous avons réalisé plusieurs cellules de mesure. Leurs principales caractéristiques sont résumées dans le tableau I.

Les tolérances d'usinage sur les diamètres sont d'environ $3 / 100 \mathrm{~mm}$.

L'alumine et le graphite ont été choisis pour leur grande inertie chimique vis-à-vis du sélénium et du tellure. Ces deux matériaux ont, en outre, un coefficient de dilatation thermique faible; ceci entraine que

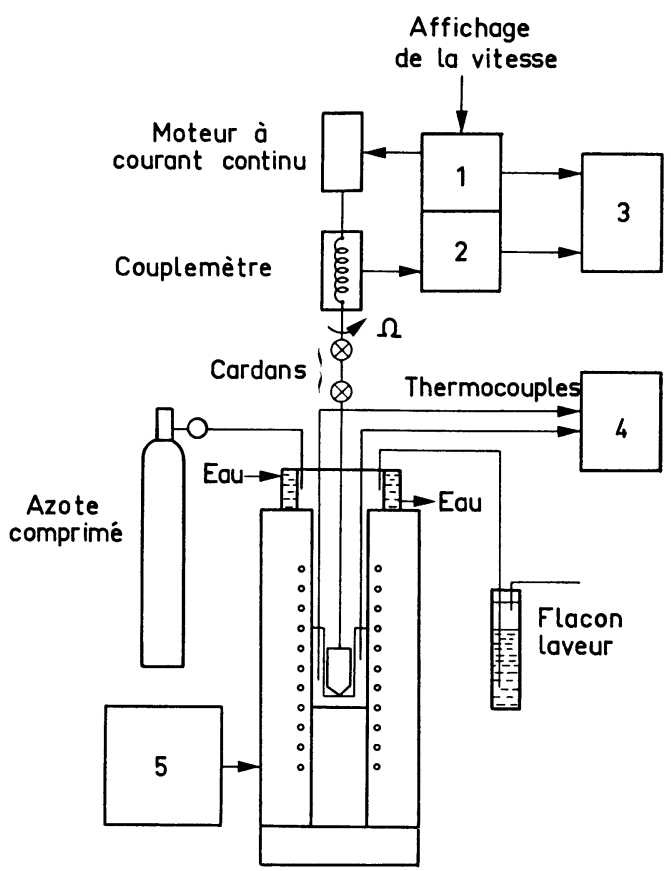

Fig. 3. - Schéma de principe du montage expérimental. 1) Alimentation du moteur et asservissement de vitesse.

2) Conversion de l'angle de torsion en signal électrique. 3) Enregistreur XY. 4) Enregistreur. 5) Régulateur du four.

l'entrefer relatif et donc le coefficient du viscosimètre varient peu avec la température. Enfin, leur utilisation rend possible le nettoyage des cellules après les mesures par l'eau régale à chaud. Compte tenu des essais que nous avons effectués, les cellules en alumine sont les mieux adaptées. En particulier leur porosité beaucoup plus faible que celle des cellules en graphite les rend imperméables au sélénium et au tellure. Cependant la facilité d'usinage du graphite nous a conduit à l'utiliser le plus souvent.

Le contact entre le rotor et le stator se fait par l'intermédiaire d'une pointe. L'utilisation à ce niveau de graphite entraînerait une usure prohibitive. Aussi, lorsque le rotor est en graphite, une pointe en alumine est rapportée à l'extrémité du cône. De même, lorsque le stator est en graphite, une pastille en alumine percée d'un trou de centrage est rapportée au fond (Fig. 1). Ainsi le contact entre le rotor et le stator se fait dans tous les cas alumine sur alumine.

TABleaU I

\begin{tabular}{|c|c|c|c|c|c|c|c|}
\hline \multirow[b]{2}{*}{ Cellule } & \multicolumn{4}{|c|}{ Rotor } & \multicolumn{3}{|c|}{ Stator } \\
\hline & Matériau & $2 R_{1}$ & $h$ & $\alpha$ & Matériau & $2 R_{2}$ & $H$ \\
\hline$\overline{\mathrm{v}}$ & - & - & - & $\overline{000}$ & - & $\overline{0}$ & $-\overline{\mathrm{mm}}$ \\
\hline 1 & Grapinte & $20,0,11110$ & & & & & \\
\hline $\mathrm{V}_{2}$ & Alumine & $40,0 \mathrm{~mm}$ & $60 \mathrm{~mm}$ & $120^{\circ}$ & Alumine & $42,0 \mathrm{~mm}$ & $110 \mathrm{~mm}$ \\
\hline $\mathrm{V}_{3}$ & Alumine & $40,0 \mathrm{~mm}$ & $60 \mathrm{~mm}$ & $120^{\circ}$ & Graphite & $42,0 \mathrm{~mm}$ & $110 \mathrm{~mm}$ \\
\hline $\mathrm{V}_{4}$ & Graphite & $41,5 \mathrm{~mm}$ & $60 \mathrm{~mm}$ & $90^{\circ}$ & Graphite & $42,0 \mathrm{~mm}$ & $110 \mathrm{~mm}$ \\
\hline
\end{tabular}


4. Dispositif expérimental. - Le viscosimètre est placé dans une enceinte en inconel (Fig. 2). Ce matériau est relativement inerte vis-à-vis des vapeurs de 'sélénium et de tellure jusqu'à $800^{\circ} \mathrm{C}$. Au-dessus du viscosimètre sont placés des bouchons en stéatite afin de diminuer les pertes calorifiques. Les vapeurs provenant du viscosimètre se condensent principalement dans la chambre aménagée dans la stéatite.

Cette enceinte est fermée par un chapeau ; il est ainsi possible de travailler sous atmosphère contrôlée. Le chapeau est muni de deux traversées étanches pour les thermocouples de mesure et de deux tubulures pour la circulation du gaz. Le mouvement de rotation est transmis par l'axe à travers une bague en bronze graphité. Une chemise d'eau maintient cette zone à basse température $\left(20\right.$ à $\left.30^{\circ} \mathrm{C}\right)$. On a ainsi un écran thermique entre le four et le couplemètre. De plus, les vapeurs non encore piégées se condensent à ce niveau.

Le four, dans lequel vient se placer l'enceinte en inconel, permet d'atteindre $800^{\circ} \mathrm{C}$ au niveau du viscosimètre avec une puissance d'environ $2 \mathrm{~kW}$.

Le schéma de principe du montage expérimental est représenté sur la figure 3 . Le dispositif comprenant le moteur et le couplemètre est un appareil Rotovisko type RV 3 (Haake). Le moteur à courant continu entraîne, par l'intermédiaire d'un ressort de torsion, l'axe de transmission. Le curseur d'un potentiomètre solidaire du ressort permet de convertir l'angle de torsion en signal électrique. La vitesse du moteur peut être réglée entre 0 et 1000 tours/mn. Deux ressorts en série de dureté différente permettent par commutation d'obtenir deux échelles de mesure $\left(0-0,5 \times 10^{-2}\right.$ $\mathrm{Nm}$ et $0-5 \times 10^{-2} \mathrm{Nm}$ ).

Entre le couplemètre et l'axe qui transmet le mouvement au rotor du viscosimètre sont placés deux cardans. Ainsi, le mouvement du rotor et la mesure du couple ne sont pas perturbés par le décalage qui peut exister entre l'axe du moteur et l'axe de transmission. En particulier, cette solution évite de fixer rigidement le moteur par rapport au viscosimètre (ou à l'enceinte en inconel).

5. Discussion de la méthode. Domaines de mesure. Au couple des forces exercées sur le rotor par le liquide et dont l'expression est donnée par l'éq. (2), doivent être ajoutés des termes correctifs. Ceux-ci correspondent aux effets d'extrémité et aux frottements parasites au niveau des centrages.

Le rotor est centré au moyen d'un trou situé au fond du stator, de l'alésage dans le bouchon en graphite et de la bague en bronze graphité (Fig. 1 et 2). A ces trois niveaux les frottements créent un couple parasite. Pour que les mesures soient significatives, il faut que celui-ci soit faible devant le couple de viscosité. Pour une cellule de dimensions données, cet impératif fixe une limite inférieure au domaine de mesures. La valeur du couple parasite est estimée pour chacune de nos cellules au moyen d'essais avec de l'eau. La différence entre le couple mesuré et celui calculé à partir de l'éq. (2) donne la limite supérieure du couple parasite. Selon les essais, le couple parasite varie entre 0 et $0,01 \times 10^{-2} \mathrm{Nm}$ pour une vitesse de rotation $\mathrm{du}$ rotor de 100 tours/mn. Aussi estimons-nous que pour être significatif le signal de mesure doit, à cette même vitesse, être supérieur à $0,1 \times 10^{-2} \mathrm{Nm}$.

Pour établir l'expression du couple en fonction de la vitesse de rotation du rotor et de la viscosité, on suppose que la répartition de la vitesse du liquide est donnée par l'éq. (1). En fait, cette hypothèse n'est vérifiée que dans la zone centrale du viscosimètre. A chaque extrémité, la répartition de la vitesse est trop complexe pour qu'on puisse calculer les forces de viscosité. Chaque cellule doit donc être étalonnée. La viscosité des huiles étalons utilisées est à $20^{\circ} \mathrm{C}$ de $1,404 \mathrm{P}$ et de $18,25 \mathrm{P}$. On constate tout d'abord que tant que la hauteur de liquide au-dessus du rotor reste inférieure à $10 \mathrm{~mm}$, celle-ci n'influe pas sur la valeur du couple mesuré. Ceci entraîne que lors des mesures il n'est pas nécessaire de contrôler ce paramètre avec précision. Par ailleurs, les essais montrent que le couple mesuré est proportionnel à la vitesse de rotation du rotor et à la viscosité. Aussi l'expression du couple peut effectivement se mettre sous la forme :

$$
C=B \eta N
$$

où $N$ est la vitesse de rotation en tours/min. et $B$ un coefficient qui ne dépend que des dimensions de la cellule. Pour la cellule $V_{2}$ (stator et rotor en alumine), le coefficient $B$ déduit des mesures a pour valeur: $(355 \pm 3) \times 10^{7}$. Si on ne tient compte que des frottements sur la partie cylindrique du rotor, le calcul conduit à $340 \times 10^{7}$. L'écart entre cette valeur et celle déterminée expérimentalement doit être associé aux frottements à chaque extrémité du rotor. Il faut noter que, même si les effets d'extrémité étaient négligeables, il serait nécessaire d'effectuer un étalonnage. En effet, avec les matériaux utilisés, les tolérances d'usinage sont de l'ordre de $3 / 100 \mathrm{~mm}$. Ceci conduit par le calcul à une précision sur $B$ d'environ $3 \%$ avec un entrefer de $1 \mathrm{~mm}$ et de $12 \%$ avec un entrefer de $0,25 \mathrm{~mm}$. L'étalonnage permet d'obtenir une précision de $1 \%$.

Nous avons vu que l'existence de couples parasites entraîne pour chaque cellule une limite inférieure du domaine de mesures. Les problèmes de la stabilité de l'écoulement imposent une limitation sur les vitesses. Tant que le nombre de Taylor associé à l'écoulement est inféi ieur au nombre critique, toute perturbation est amortie dans le temps et l'écoulement laminaire est dit stable. Le temps nécessaire à l'amortissement d'une perturbation est d'autant plus grand que le nombre de Taylor est voisin du nombre critique ; lorsque la durée entre deux perturbations est inférieure au temps de relaxation, l'écoulement n'est jamais stationnaire. L'enregistrement du couple présente alors des oscillations autour d'une valeur moyenne. L'amplitude de ces oscillations est d'autant plus grande que la fréquence 
TABLEAU II

\begin{tabular}{|c|c|c|c|c|c|c|}
\hline \multirow[b]{2}{*}{ Cellules } & \multirow{2}{*}{$\begin{array}{c}\text { Coefficient de la } \\
\text { cellule } \\
B=C / N \eta \\
C \text { en } \mathrm{N} . \mathrm{m} \\
N \text { en tours } / \mathrm{min} . \\
\eta \text { en poises }\end{array}$} & \multicolumn{2}{|c|}{$\begin{array}{l}\text { Limite supérieure du } \\
\text { domaine de mesures } \\
\left(C=5 \times 10^{-2} \mathrm{~N} . \mathrm{m}\right) \\
(N=25 \text { tours/min.) }\end{array}$} & \multicolumn{3}{|c|}{$\begin{array}{c}\text { Limite inférieure du domaine de mesures } \\
\left(C=0,1 \times 10^{-2} \mathrm{~N} . \mathrm{m}\right) \\
(N=100 \text { tours } / \mathrm{min} .)\end{array}$} \\
\hline & & $\eta(\mathrm{Po})$ & Précision & $\eta(\mathrm{Po})$ & Précision & $\begin{array}{c}\text { Limite supérieure } \\
\text { de la vitesse } \\
\text { de rotation } \\
\left(N \leqslant N_{\mathrm{c}} / 2\right) \\
\rho=5 \mathrm{~g} / \mathrm{cm}^{3}\end{array}$ \\
\hline $\mathrm{V}_{1}$ & $221 \times 10^{7}$ & 1000 & $1 \%$ & 5 & $10 \%$ & 1000 tours/min. \\
\hline$V_{2}-V_{3}$ & $355-342 \times 10^{7}$ & 50 & $1 \%$ & 0,25 & $10 \%$ & 250 tours $/ \mathrm{min}$ \\
\hline $\mathrm{V}_{4}$ & $2080 \times 10^{7}$ & 10 & $1 \%$ & 0,05 & $10 \%$ & 350 tours $/ \mathrm{min}$ \\
\hline
\end{tabular}

des perturbations est élevée et que le nombre de Taylor est proche du nombre critique. Expérimentalement, on constate que pour des vitesses de rotation inférieures à la moitié de la vitesse critique l'amplitude des oscillations ne dépasse jamais $5 \%$ de la valeur du signal (les constantes de temps du détecteur étant d'environ $10 \mathrm{~s}$ ). Lors des mesures, nous avons pris comme règle de ne pas dépasser ce taux d'oscillations. Ceci conduit pour chaque cellule à limiter les vitesses de rotation à la moitié des vitesses critiques associées à l'écoulement dans la zone centrale (tableau II). En fait, à ces vitesses, il est probable que des instabilités de Taylor se développent à chaque extrémité du rotor. Cependant la contribution de ces écoulements au couple de frottement est faible et la mesure n'est pas perturbée par ces instabilités. Ainsi, pour les différentes cellules utilisées on constate que le couple reste proportionnel à la vitesse de rotation jusqu'à des vitesses proches des vitesses critiques calculées. L'existence de ces instabilités a comme action principale d'augmenter la fréquence des perturbations et ainsi d'entretenir les oscillations.

Le couple de frottements augmente proportionnellement à la viscosité. Le détecteur ne permettant pas de mesurer des couples supérieurs à $5 \times 10^{-2} \mathrm{~N}$.m on est amené aux fortes viscosités à réduire le domaine d'exploration en vitesse. Il s'ensuit que la précision diminue. Aussi, pour chaque cellule, nous avons considéré que la valeur maximale de la viscosité mesurable est telle que le domaine de vitesses ne soit pas inférieur à 0-25 tours/min. La précision est alors d'environ $1 \%$.

Sur le tableau II, sont regroupées les caractéristiques des quatre cellules que nous avons réalisées.

6. Viscosité de sélénium. - Avant d'étudier les alliages Se-Te, nous avons voulu nous assurer de la validité de notre méthode de mesure. Aussi avons-nous déterminé la viscosité du sélénium et comparé nos résultats à ceux obtenus par différents auteurs, $[5,6$, $7,8,9]$.
Le sélénium est introduit dans les cellules sous formes de granules. La masse utilisée est telle qu'au point de fusion la hauteur de liquide au-dessus du rotor soit d'environ $5 \mathrm{~mm}$. Les mesures sont effectuées sous azote, la surpression étant d'environ $10^{4}$ pascals.

Lors de chaque mesure, la température est stabilisée à $\pm 1^{\circ} \mathrm{C}$. Le gradient de température entre les deux extrémités du rotor est toujours inférieur à $1^{\circ} \mathrm{C}$. Ainsi, la précision sur la température peut être évaluée à $2{ }^{\circ} \mathrm{C}$. Pour chaque palier de température, le couple est mesuré en fonction de la vitesse, les points de mesure étant relevés après stabilisation de l'écoulement.

6.1 ETUde DE LA VISCOSITÉ EN FONCTION DU GRADIENT DE VITESSE. - Le sélénium liquide, constitué d'anneaux et de chaînes relativement longues [7], [10], aurait pu présenter un caractère non newtonien. En fait, nos mesures montrent que, dans toute la gamme de température étudiée $\left(211-625^{\circ} \mathrm{C}\right)$, le couple est toujours proportionnel au gradient de vitesse et donc que le sélénium se comporte comme un liquide newtonien. Sur la figure 4 sont portées les courbes obtenues avec la cellule $V_{1}$; ces résultats sont les plus significatifs. En effet, le caractère non newtonien du sélénium devrait être d'autant plus marqué que la longueur des chaînes est grande et donc que la température est basse. De plus, la cellule $V_{1}$ est celle qui permet pour des températures voisines du point de fusion

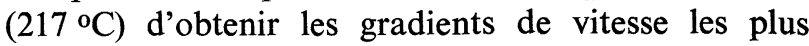
élevés et les contraintes les plus grandes (de l'ordre de $4 \times 10^{3} \mathrm{~N} / \mathrm{m}^{2}$ ). Enfin les vitesses critiques de rotation étant très largement supérieures à 1000 tours/min., toute non linéarité observée peut être attribuée au caractère non netwonien du liquide. Or, on constate d'après les courbes de la figure 4 que le couple mesuré est proportionnel au gradient de vitesse. Ceci entraîne que l'hypothèse du caractère non newtonien du sélénium doit être rejetée tout au moins dans la phase liquide et dans la gamme des contraintes étudiées. En effet, dans la phase solide, Cukierman a mis en évidence 


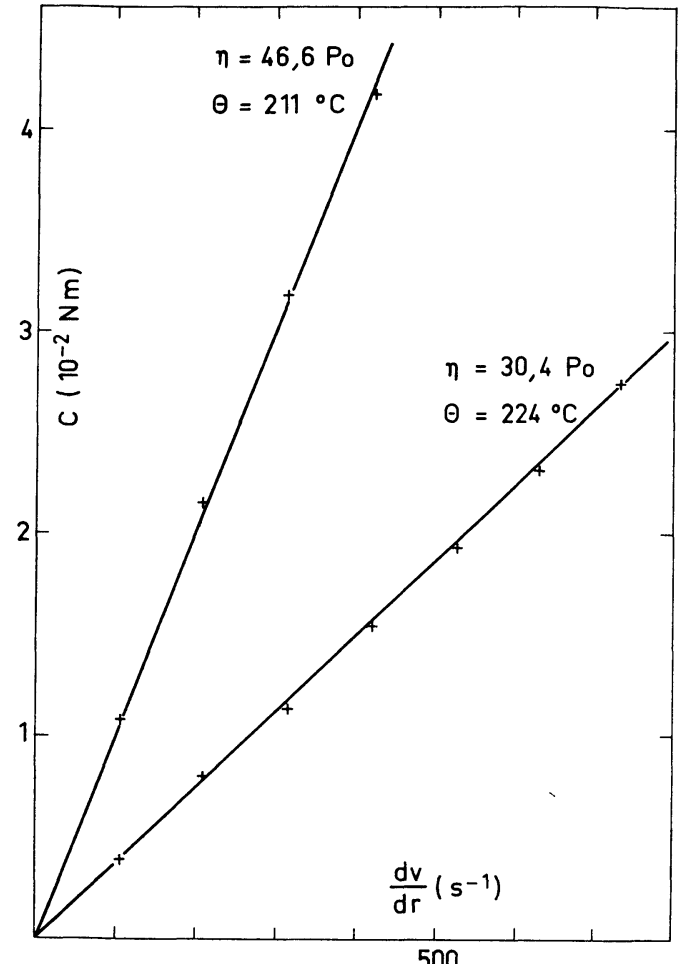

Fig. 4. - Couple exercé sur le rotor en fonction du gradient de vitesse.

que le sélénium amorphe est non newtonien; la viscosité est alors de l'ordre de $10^{12} \mathrm{P}$ tandis que les contraintes sont d'environ $10^{7} \mathrm{~N} / \mathrm{m}^{2}$ [6].

6.2 ETUdE DE LA VISCOSITÉ EN FONCTION DE LA TEMPÉRATURE. - Le point de fusion du sélénium est de $217^{\circ} \mathrm{C}$. Avec les cellules utilisées, il est possible de maintenir le liquide en surfusion jusqu'à $211^{\circ} \mathrm{C}$. La

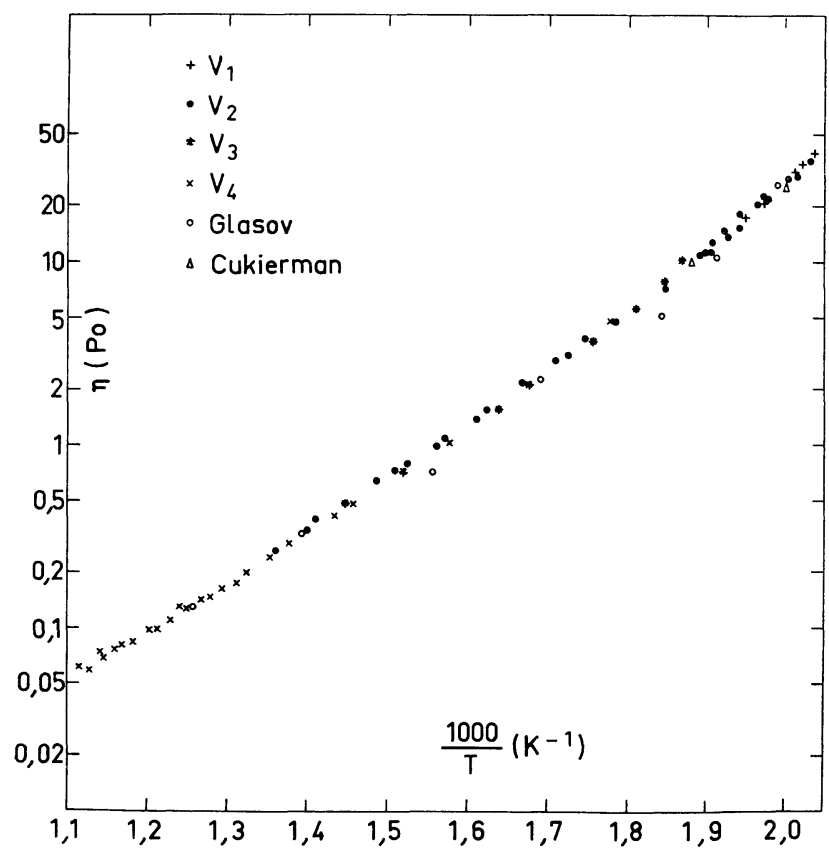

Fig. 5. - Viscosité dynamique du sélénium. gamme étudiée s'étend de cette température jusqu'à $625^{\circ} \mathrm{C}$. Au-dessus, la viscosité devient trop faible pour être mesurée. Sur la figure 5 est portée sur une échelle semi-logarithmique la viscosité exprimée en poises en fonction de l'inverse de la température absolue. Pour chaque point nous avons vérifié que le couple est proportionnel à la vitesse. On constate que les résultats obtenus pour chaque cellule se recoupent avec une bonne précision. Ainsi les mesures ne sont affectées ni par la nature des matériaux utilisés pour réaliser les cellules ni par leurs dimensions géométriques. Sur cette même figure, nous avons porté les résultats obtenus par Glasov [5] et Cukierman [6]. L'accord est satisfaisant compte tenu de la précision et du fait que les méthodes de mesure sont différentes. D'autres résultats expérimentaux sont également en bon accord avec ce travail, $[7,8,9]$. Ceci nous conduit à conclure à la validité de notre dispositif expérimental.

7. Conclusion. - La principale limitation de la méthode utilisée est due à la difficulté de mesurer les faibles viscosités (inférieures 0,05 P). En théorie, ces mesures sont possibles; en fait cela implique des problèmes mécaniques très difficiles à résoudre à haute température et avec des liquides corrosifs. La simplicité de mise en œuvre d'autres méthodes (méthode du capillaire par exemple) compense alors leurs inconvénients.

Hormis le domaine des faibles viscosités, l'utilisation de viscosimètres rotatifs présente de nombreux avantages. Tout d'abord, ils permettent avec une seule cellule de couvrir une grande étendue de mesures. Ainsi, malgré nos critères très restrictifs, chacune de nos cellules permet de déterminer des viscosités dans un rapport 1 à 200. La méthode du pendule oscillant qui permet de couvrir un domaine aussi étendu nécessite d'utiliser des formules différentes et donc un étalonnage différent selon le nombre de Reynolds associé à l'écoulement [11].

Le second intérêt est que cette méthode est la seule qui permette de s'assurer que le liquide étudié est newtonien et d'exploiter les résultats lorsqu'il ne l'est pas. Ainsi nous avons pu vérifier que le sélénium est un liquide newtonien alors qu'il était possible, compte tenu de sa structure, d'envisager le cas contraire.

Enfin, l'utilisation des viscosimètres rotatifs permet de contrôler à tout moment le régime de l'écoulement. Ce point est essentiel. En effet, prenons le cas du sélénium. Sa viscosité qui est au point de fusion d'environ $40 \mathrm{P}$, décroît avec une énergie d'activation de $0,72 \mathrm{eV}$ jusqu'à $280^{\circ} \mathrm{C}$ où une cassure est observée. Entre $280^{\circ} \mathrm{C}$ et $500^{\circ} \mathrm{C}$, la viscosité décroît avec une énergie d'activation de $0,60 \mathrm{eV}$. A $500^{\circ} \mathrm{C}$, une seconde cassure est observée ; au-dessus de cette température, l'énergie d'activation est de $0,50 \mathrm{eV}$. Le fait d'avoir contrôler l'écoulement nous permet d'associer ces cassures à des variations structurales du liquide et non pas à l'établissement de turbulences. Dans les autres 
méthodes, il est nécessaire pour vérifier ce point d'utiliser plusieurs cellules afin d'obtenir des gradients de vitesse différents. Il faut noter que les cassures observées se retrouvent dans l'étude en fonction de la température d'autres propriétés physiques telles que la conductivité électrique en champ faible et en champ fort, la photoconductivité...

Actuellement, nous poursuivons ces travaux par la mesure de la viscosité des alliages Se-Te. Ceci devrait nous permettre de préciser l'influence de la longueur des chaînes et du nombre d'anneaux sur les cassures observées.

En conclusion, on peut dire que ce viscosimètre est un outil de travail puissant, particulièrement bien adapté pour les liquides à forte viscosité. Une extension à de plus hautes températures est possible par l'emploi de matériaux réfractaires adaptés. Cependant le fait de ne pas pouvoir mesurer les viscosités inférieures à $0,05 \mathrm{P}$ interdit son utilisation dans le cas des métaux liquides et constitue une limite à notre appareillage.

\section{Bibliographie}

[1] LANDAU, L., Lifchitz, E., Mécanique des Fluides (Editions Mir, Moscou) 1971.

[2] Lord Rayleigh, Sci. Pap. 6 (1920) 447.

[3] TAYlor, G. I., Philos. Trans. R. Soc. 223A (1923) 289.

[4] Chandrasekhar, S., Hydrodynamic and Hydromagnetic stability (Oxford University Press, Londres) 1961.

[5] Glasov, V. H., Chizhevskaya, S. N., Glagoleva, N. N., Liquid Semiconductors (Plenum Press, New York) 1969.

[6] Cukierman, M., Uhlmann, D. R., J. Non-Cryst. Solids 12 (1973) 199.
[7] Keezer, R. C., Griffiths, C. H., Vernon, J. P., J. Cryst. Growth 3-4 (1968) 755.

[8] Hamada, S., Yoshida, N., Shirai, T., Bull. Chem. Soc. Japan 42 (1969) 1025.

[9] FisCHER, M., KREBS, H., Glastechn. Ber. 47 (1974) 42.

[10] Siemsen, K. J., Riccius, H. D., J. Phys. Chem. Solids 30 (1969) 1897.

[11] ShvidxovskiI, E. G., Some Problems in Viscosity of Molten Metals (Gostekhteorizdat, Moscou) 1955. 